\title{
BMJ Open Anticipatory guidance to prevent infant sleep problems within a randomised controlled trial: infant, maternal and partner outcomes at 6 months of age
}

\author{
Barbara C Galland, ${ }^{1}$ Rachel M Sayers, ${ }^{1}$ Sonya L Cameron, ${ }^{1}$ Andrew R Gray, ${ }^{2}$ \\ Anne-Louise M Heath, ${ }^{3}$ Julie A Lawrence, ${ }^{1}$ Alana Newlands, ${ }^{3}$ Barry J Taylor, ${ }^{1}$ \\ Rachael W Taylor ${ }^{4}$
}

To cite: Galland BC, Sayers RM, Cameron SL, et al. Anticipatory guidance to prevent infant sleep problems within a randomised controlled trial: infant, maternal and partner outcomes at 6 months of age. BMJ Open 2017;7:e014908. doi:10.1136/ bmjopen-2016-014908

- Prepublication history and additional material are available. To view please visit the journal online (http://dx.doi.org/10. 1136/bmjopen-2016-014908).

Received 27 October 2016 Revised 31 March 2017 Accepted 12 April 2017

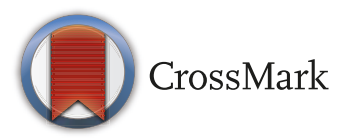

${ }^{1}$ Department of Women's and Children's Health, University of Otago, Dunedin, New Zealand ${ }^{2}$ Department of Preventive and Social Medicine, University of Otago, Dunedin, New Zealand ${ }^{3}$ Department of Human Nutrition, University of Otago, Dunedin, New Zealand

${ }^{4}$ Department of Medicine, University of Otago, Dunedin, New Zealand

Correspondence to Dr Barbara C Galland; barbara.galland@otago.ac.nz

\section{ABSTRACT}

Objective To evaluate the effectiveness of sleep education delivered antenatally and at 3 weeks postpartum to prevent infant sleep problems at 6 months of age.

Design Sleep intervention within a randomised controlled trial for the Prevention of Overweight in Infancy (POI) study.

Participants 802 families were randomly allocated to one of four groups: usual care (control), sleep intervention (sleep), food, activity and breastfeeding intervention (FAB), and combined group receiving both interventions (combination).

Interventions All groups received standard Well Child care. The sleep intervention groups (sleep and combination) received an antenatal group education session (all mothers and most partners) emphasising infant self-settling and safe sleeping, and a home visit at 3 weeks reinforcing the antenatal sleep education. $\mathrm{FAB}$ and combination groups received four contacts providing education and support on breast feeding, food and activity up to 4 months postpartum.

Outcome measures Here we report secondary sleep outcomes from the $\mathrm{POI}$ study: the prevalence of parentreported infant sleep problems and night waking, and differences in sleep duration. Additional outcomes reported include differences in infant self-settling, safe sleep practices, and maternal and partner reports of their own sleep, fatigue and depression symptoms.

Results Linear or mixed linear regression models found no significant intervention effects on sleep outcomes, with $19.1 \%$ of mothers and $16.6 \%$ of partners reporting their infant's sleep a problem at 6 months. Actigraphy estimated the number of night wakings to be significantly reduced $(8 \%)$ and the duration of daytime sleep increased $(6 \mathrm{~min})$ in those groups receiving the sleep intervention compared with those who did not. However, these small differences were not clinically significant and not observed in 24 hours infant sleep diary data. No other differences were observed.

Conclusion A strategy delivering infant sleep education antenatally and at 3 weeks postpartum was not effective in preventing the development of parent-reported infant sleep problems.

\section{Strengths and limitations of this study}

- The first randomised controlled trial examining the efficacy of a sleep education programme to prevent infant sleep problems commencing antenatally.

- Data were collected from both mother and partner about whether their child's sleep was a problem and partners as well as mothers rated their own sleep quality across several time points.

- Sleep data from infants were reported both subjectively (sleep diaries) and objectively (actigraphy)

- Key education outcomes related to infant sleep practices, such as infant self-settling, were collected via parent report and may not be accurate enough to identify any intervention effects.

- The results are limited to young infants in a welleducated community, which may reduce the generalisability of the findings.

\section{INTRODUCTION}

Unsettled sleep-wake behaviour peaks in the first 6 months of life, with between $16 \%$ and $38 \%$ of parents reporting sleep problems to clinicians within the first year. ${ }^{1-3}$ Parents' most common concerns relate to their baby's crying, ${ }^{4}$ amount of sleep, appropriate strategies to settle their infant to sleep, ${ }^{56}$ difficulties settling to sleep and frequent night waking. ${ }^{7}$ Infant sleep problems are expensive to treat $^{8}$ and are associated with several adverse consequences for mothers, including postnatal depression, ${ }^{8}$ fatigue, ${ }^{9}$ as well as poorer general and mental health among those without a history of depression. ${ }^{10}$ Although fathers have rarely been studied, they also report poorer general health and greater psychological distress linked to infant sleep problems. ${ }^{10}$

Infant sleep problems likely emerge from interactions between infant and parental health and behaviour, and the 
environment. ${ }^{11}$ One third of infants who are unable to achieve a consolidated sleep of 6 hours by 5 months of age still cannot achieve this by $2^{1 / 2}$ years of age, ${ }^{12}$ and a short sleep duration pattern sustained across infancy and early childhood is associated with five times greater odds of children exhibiting hyperactive behaviour at 5 years of age. ${ }^{7}$ Evidence also suggests that sleep problems first presenting in infancy are likely to persist into childhood, ${ }^{13-15}$ with potential for poorer cognitive development, ${ }^{16-18}$ behaviour ${ }^{16}{ }^{19}$ and quality of life, ${ }^{20}$ and a greater risk of overweight or obesity. ${ }^{21-24}$ These adverse outcomes highlight the need for early intervention to optimise sleep development.

Overall, interventions designed to treat sleep problems once they have become established have demonstrated a small to medium effect on increasing infant nocturnal total sleep time, ${ }^{25}$ either no or small effects on reducing night wakings, ${ }^{25}{ }^{26}$ and small impacts on improving maternal mood, ${ }^{25}$ depression, fatigue, sleep quality and maternal cognitions about their infant's sleep. ${ }^{26}$ Whether prevention of sleep problems might be more effective, and potentially less costly, than attempts to treat established issues is largely unknown. Randomised controlled trials (RCTs) emphasising the early establishment of positive sleep practices have produced mixed findings to date. ${ }^{27-29}$ These interventions were delivered postnatally ${ }^{28} 29$ or at 3 months postpartum, ${ }^{27}$ and on a one-to-one basis. None were delivered antenatally or within group sessions where families may be less stressed and more receptive to the sleep education messages. Given that a large number of infants suffer sleep problems and that the flow-on effects of these problems carry significant long-term costs to individuals, it is pertinent to investigate the effectiveness of simple prevention strategies that could be implemented as a public health measure through antenatal education and Well Child services.

We recently undertook a four-armed RCT, the Prevention of Overweight in Infancy (POI) study ${ }^{30}$ to determine whether additional support and education on food, activity, breast feeding and sleep from late pregnancy could reduce overweight and obesity in children at 2 years of age. In exploratory analyses, the trial found a protective effect for obesity among groups receiving the sleep intervention. ${ }^{31}$ The exact mechanisms by which sleep could influence obesity in early life are unclear, although the ability of an infant to learn to self-regulate his/her own sleep is considered one of the earliest biological markers of self-regulatory behaviours. ${ }^{32}$ This enhanced sleep self-regulation could conceivably help shape other self-regulatory behaviours linked to healthy weight. Other potential mechanisms linked to insufficient sleep in children and adults include changes in neurohormonal control of appetite regulation, impacts on dietary intake and increased sedentary activity — all favouring weight gain. ${ }^{33}$

The aim of this paper was to determine whether the infant sleep education programme delivered antenatally and 3 weeks postpartum reduced the prevalence of parent-reported sleep problems and night wakings, and influenced sleep duration in infants up to 6 months of age (secondary outcomes of the RCT; ClinicalTrials. gov: NCT00892983). Other aims were to determine any differences in infant self-settling and safe sleep practices, and maternal and paternal reports of their own sleep, fatigue and depression symptoms (additional outcomes).

\section{METHODS}

Study design

The POI study (Clinical Trials NCT00892983) was a fourarmed RCT commencing in late pregnancy. Data from baseline (third trimester) to 6 months of age are reported here.

\section{Participants and recruitment}

All mothers booked into the single maternity hospital ( $>97 \%$ of all births) serving the city of Dunedin between May 2009 and November 2010 were potentially eligible. Letters of invitation were sent to mothers at 28-30 weeks' gestation and an 'opt out' recruitment strategy was used. Exclusion criteria applied before birth were home address outside the greater Dunedin area, planning to move away in the next 2 years, not booked with the maternity centre or homebirth midwife before 34 weeks' gestation, or unable to communicate in English or Te Reo Māori (language of the indigenous people of New Zealand). Exclusion criteria applied after birth were identification of a congenital abnormality likely to affect feeding or growth, or the infant being born before 36.5 weeks' gestation.

\section{Randomisation}

Participants were randomised to one of four groups: (1) usual care (control), (2) food, activity and breastfeeding intervention (FAB), (3) sleep intervention (sleep), or (4) combined intervention group receiving both the sleep and FAB interventions (combination). Details of allocation concealment and stratification have been described previously, ${ }^{30}$ but briefly stratification was based on household deprivation (tertiles: low (NZ Deprivation Index deciles 1-3), ${ }^{34}$ medium (4-7) and high (8-10)) and parity including the study child ( 1 vs $\geq 2)$. Group allocation was revealed to the participants after they consented to participate. The statistician remained blinded to group allocation codes until primary analyses were completed.

\section{Usual care}

Families of all four groups received standard maternity and Well Child care from a maternity care professional and a Well Child provider of their choice. Well Child care $^{35}$ aims to maximise the health and development of all New Zealand children up to age 5 years (on average 8-9 visits in the first 6 months of life). The control group received this usual care only. 


\section{Interventions}

\section{Sleep}

Families receiving the sleep intervention (sleep and combination groups) received antenatal education delivered via a single group session (up to 1 hour), followed by one home visit at 3 weeks postpartum with a researcher with infant sleep training. Antenatal sessions educated all mothers and most partners about normal developmental patterns of infant sleep and emphasised infant settling to sleep unaided (ie, self-settling or self-regulation of sleep), which appears to be a key factor in the development of healthy sleep-wake patterns and a precursor to longer sleep duration in later infancy, ${ }^{36}$ and safe sleep practices as measures to prevent sudden unexpected death in infancy ${ }^{37}$ (summarised in online supplementary appendix 1). Partners were mostly fathers. Parents left equipped with a 'tip sheet' related to the key education messages. The individual sessions at the 3-week home visit reinforced the antenatal sleep education and were conducted with the aid of a more detailed 15-page booklet covering the key messages for developing healthy sleep and safe sleep practices (summarised in online supplementary appendix 2). At this visit, researchers provided advice on any concerns the families had with their infant's sleep, and parents were encouraged to keep using the booklet as a valuable resource and to contact research staff at any stage should they require further sleep advice.

\section{FAB}

Mothers in the FAB group received three contacts providing education and support on breast feeding (antenatal, 1 week and 4 months postpartum) and one on physical activity (3months postpartum) before 6 months of age. An international board-certified lactation consultant worked with each family to assist with infant feeding, focusing on prolonging exclusive breast feeding and delaying the introduction of complementary foods until around 6 months of age. At 3 months, each family attended an Active Movement session delivered by an established local sports body. The FAB intervention is described in more detail elsewhere. ${ }^{38}$

\section{Combination}

This group received five intervention visits in total before 6 months of age as the FAB and sleep antenatal session was combined.

\section{Questionnaires}

Parents completed questionnaires during home visits at baseline (third trimester, prior to allocation) and at 4 and 6 months. Questionnaires collected data relevant to outcomes of the sleep education, including ratings of the baby's problematic sleep and unsettled behaviour, and of infant settling and safe sleep practices. Mothers and partners also rated perceptions of their own sleep quantity and quality, and reported the usual time they went to bed, usual sleep time (sleep onset) and usual wake time (sleep offset). Their sleep duration was calculated as the difference between sleep onset and sleep offset. Sleep latency (time taken to fall asleep) was calculated from the difference between the time they went to bed and sleep onset. Ratings of maternal and partner fatigue and depression symptoms (Edinburgh Postnatal Depression Scale; $\operatorname{EPDS}^{39}$ ) were also collected. Duration of exclusive breast feeding (ie, the infant receiving no liquids, other than breast milk, and no solids since birth) was calculated as age to the nearest part week from the monthly questionnaires. $^{30}$

\section{Sleep diary}

When the infant was 6 months of age, parents were asked to complete a 48-hour diary during the 5-day to 7-day period when actigraphy was also recorded (as described previously ${ }^{40}$ ). Time was displayed horizontally in $5 \mathrm{~min}$ grid blocks. A parent was asked to indicate using an arrow the time the infant went to sleep and awoke, including daytime naps. Grid block counts with sleep and wake coding were then entered into a spreadsheet set up to automatically calculate standard sleep-wake variables.

\section{Actigraphy}

Infants wore an Actical accelerometer (Mini-Mitter, Bend, Oregon, USA) for 5-7 days fitted on the shin. The accelerometers were initialised using $15 \mathrm{~s}$ epochs. Sleep onset (evening sleep time) and morning sleep offset (wake time) events were ascertained visually from activity graphs and marked in the sleep scoring programme in MATLAB (MathWorks, Natick, Massachusetts, USA), from which standard sleep-wake variables, including nap counts and nap duration, were determined using the count-scaled algorithm. A sleep period was defined as at least $20 \mathrm{~min}$ of sleep preceded by $5 \mathrm{~min}$ of awake, with the sleep period altered for nap detection between 09:00 and 17:00 as previously described using a minimum nap time threshold of 20 min. ${ }^{40}$

\section{Statistical analysis}

The RCT was powered on detecting differences in growth (the primary outcome) between the arms of the study ${ }^{30}$; therefore, no relevant sample size calculations are available. The prevalence of parent-reported infant sleep problems, the number of night wakings and sleep duration at 6 months of age are secondary outcomes of the RCT as reported here. All other outcomes reported are considered additional outcomes of the RCT. The data were analysed using Stata V.14.1. Statistical significance was determined by two-sided $\mathrm{p}<0.05$ in all cases. No adjustments were made for multiple comparisons. Continuous outcome variables were compared between the four groups using linear regression models where the outcome was at a single time point and mixed linear regression with a random participant effect where multiple time points were included. Where model residuals were skewed or demonstrated heteroscedasticity, natural log transformations were investigated, after adding one for variables including 0 values. If issues with model residuals were not 
thereby resolved, quantile regression was used instead to model medians. All models included the stratification variables (three levels of household deprivation and two levels of parity).$^{41}$ Binary variables were similarly analysed using logistic regression and mixed logistic regression with a random participant effect. For all outcomes, a second set of models looking at the main effects of the sleep (sleep and combination groups vs FAB and control) and FAB interventions (FAB and combination groups vs sleep and control) were implemented providing there was no evidence of an interaction between the two interventions. The purpose of this two-step process was to maximise the power to detect main effects but, as is well known, ${ }^{42}$ this increases type I error rates above their nominal level, and so these results should be interpreted with caution, especially in cases of marginal statistical significance.

\section{RESULTS}

\section{Participant characteristics at baseline}

Of the $847(58 \%)$ eligible families who agreed to participate, 802 met the postpartum inclusion criteria. Table 1 presents participant characteristics, illustrating that the majority of mothers and infants were European and $48 \%$ of mothers were having their first baby. Participating mothers had a mean age of 32 years and were well educated, with $65 \%$ having completed a university degree. The majority of EPDS scores were within the range of 'normal' for mothers ${ }^{43}$ and fathers. ${ }^{44}$ Participant flow through the study has been described elsewhere. ${ }^{38}$

\section{Parental sleep at baseline}

Mothers reported obtaining approximately 8.4 hours of sleep at baseline and partners 7.6 hours (table 2). Approximately $65 \%$ of mothers reported taking $30 \mathrm{~min}$ or longer to get to sleep (long sleep latency) and 58\% of partners. Self-ratings of sleep quantity and quality for both mothers and partners were in the mid-range of a 1-8 rating scale, where higher scores reflected better satisfaction with sleep quantity and better sleep quality. Categorising the satisfaction ratings by poor versus good sleep quantity or quality (ratings $\leq 3 \mathrm{vs}>3$ ) led to $29 \%$ and $21 \%$ of mothers reporting poor sleep quantity and quality, respectively. For partners poor sleep quantity and quality were reported by $14 \%$ and $8 \%$, respectively.

\section{Outcome measures}

\section{Infant sleep}

Analyses of sleep diary data available at 6 months from 507 infants (table 3) demonstrate there were no significant intervention effects related to parent-reported number of night wakings, 24-hour or overnight sleep duration, longest sleep duration, sleep efficiency, or the number of daytime naps. Actigraphy data provided from 498 infants recorded significantly fewer episodes of night waking and longer daytime sleep for infants in the groups receiving sleep interventions (sleep and combination groups) compared with the groups who did not receive the sleep intervention (control and FAB groups). However the number of night wakings was reduced by $8 \%$ and the sleep duration increase was only approximately $6 \mathrm{~min}$. No other significant intervention effects were found for the actigraphy variables. Exclusive breastfeeding duration (median 13-17 weeks) did not differ across the groups $(p \geq 0.323)$ and thus was not considered to be a covariate that might influence night waking.

\section{Problematic sleep}

There were no intervention effects on parental reports that their infant's sleep was problematic (table 4). In total, $16.1 \%$ (4 months) and $19.9 \%$ (6 months) of mothers reported that their infant's sleep was a problem (rated as 5-8 on an 8-point scale). In partners (mostly fathers), the prevalence was $11.7 \%$ (4 months) and $16.6 \%$ (6 months). Interestingly, there was a lack of agreement between mothers and partners about whether or not their baby's sleep was a moderate to large problem (kappa 0.25 and 0.29 , respectively). At 4 months there was no evidence of a difference between mothers and partners, but at 6 months mothers were significantly more likely $(p=0.050)$ to be the one reporting problems among the 94 discordant pairs (57 vs 37 ).

\section{Settling practices}

Practices relevant to sleep education on settling babies were not influenced by the interventions (table 4), although there was a non-statistically significant tendency for groups who had received the sleep education (sleep and combination groups) to more frequently put their baby to sleep awake $(\mathrm{p}=0.052)$. Overall more than half of the mothers reported high scores (6-7 on a scale of $1-7)$ for the frequency of putting their baby to sleep awake $(54.2 \%$ and $60.3 \%$ at 4 and 6 months, respectively), letting their baby fall asleep without them being present $(51.6 \%$ and $56.8 \%$ at 4 months and 6 months, respectively), and the majority reported being frequently able to recognise when their baby was tired $(77.9 \%$ at 4 months and $83.2 \%$ at 6 months). A small intervention effect was found for the time it took babies to go to sleep (sleep latency; $\mathrm{p}=0.038$ ), with significant differences in the prevalence of those reporting their infant to usually take $\geq 30$ min (ie, a long sleep latency) to fall asleep at 6 months $(\mathrm{p}=0.015)$. This was attributed to fewer infants in the combination group (who received sleep education) having long sleep latencies.

\section{Safe sleep practices}

Practices relevant to safe sleep messages were not influenced by the interventions (table 4). Regular bedsharing was only practised by a minority: overall $6.4 \%$ at 6 months. The majority of infants slept on their back, following recommended practices, and few families smoked in the car while children were present or smoked in the household, with numbers too low for analyses. Data from the control arm of the study for these safe sleep variables at infant ages 3-23 weeks have been published previously. ${ }^{37}$ 
Table 1 Infant, maternal and partner characteristics

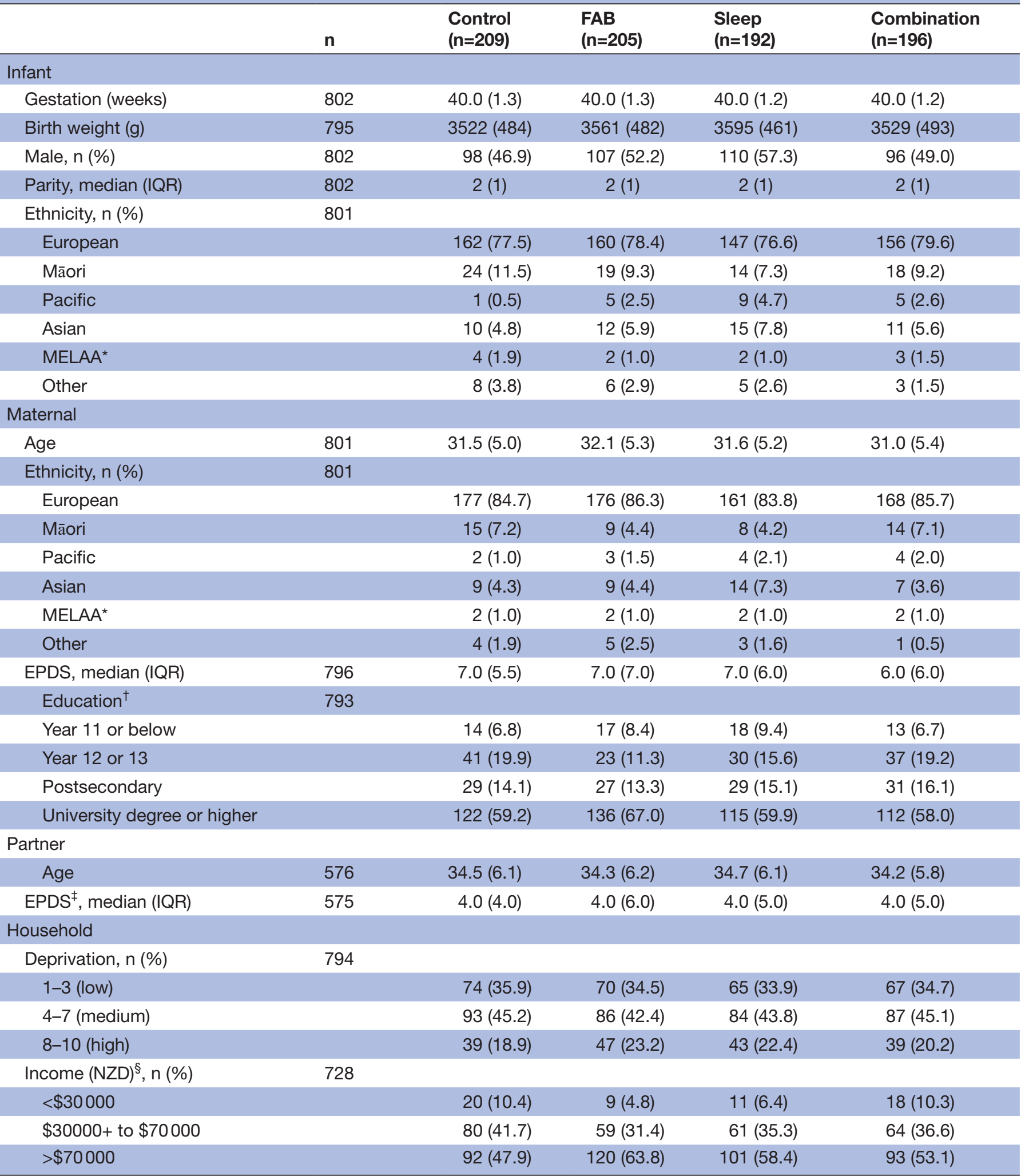

Data presented as mean (SD) unless stated otherwise.

*MELAA refers to Middle Eastern, Latin American or African.

†Secondary schooling in New Zealand is from year 9 to 13 inclusive; postsecondary qualifications refer to all those that are non-university based.

‡EPDS, Edinburgh Postnatal Depression Scale (0-30); higher score is worse.

$\S N Z D$, New Zealand dollars. $\$ 70000$ represents average annual household income in the region at the time of study.

FAB, food, activity and breastfeeding intervention. 
Table 2 Parental sleep at baseline (third trimester)

\begin{tabular}{|c|c|c|c|c|c|}
\hline & $\mathbf{n}$ & Control $(n=209)$ & FAB $(n=205)$ & Sleep $(n=192)$ & Combination $(n=196)$ \\
\hline \multicolumn{6}{|l|}{ Mother } \\
\hline Sleep duration (hour) & 797 & $8.3(1.1)$ & $8.4(1.2)$ & $8.4(1.0)$ & $8.3(1.4)$ \\
\hline Sleep Quantity Score* & 802 & $4.4(1.3)$ & $4.3(1.4)$ & $4.2(1.3)$ & $4.4(1.4)$ \\
\hline Poor score, n (\%) & & $54(26)$ & $63(31)$ & $61(31)$ & $53(27)$ \\
\hline Poor score, n (\%) & & $35(17)$ & $45(22)$ & $40(21)$ & $42(22)$ \\
\hline \multicolumn{6}{|l|}{ Partner } \\
\hline Sleep duration (hour) & 590 & $7.6(0.9)$ & $7.6(0.9)$ & $7.6(0.9)$ & $7.7(1.0)$ \\
\hline Sleep latency $\geq 30 \mathrm{~min}, \mathrm{n}(\%)$ & 548 & $88(58)$ & $89(61)$ & $82(61)$ & $69(52)$ \\
\hline Sleep Quantity Score* & 577 & $4.0(1.2)$ & $3.8(1.3)$ & $3.9(1.2)$ & $4.0(1.3)$ \\
\hline Poor score, n (\%) & & $6(4)$ & $12(8)$ & $12(9)$ & $13(9)$ \\
\hline
\end{tabular}

Data presented as mean (SD) unless stated otherwise.

*From a possible score of 1 (not nearly enough) to 8 (more than enough), thus higher score is better, poor score $\leq 3$.

†From a possible score of 1 (very bad) to 8 (very good), thus higher score is better at baseline, poor score $\leq 3$.

$\mathrm{FAB}$, food, activity and breastfeeding intervention.

\section{Maternal and partner outcomes}

At infant age 6 months, there were no differences between intervention groups for maternal or partner sleep quantity, their sleep-rated quality nor the prevalence of long sleep latency ( $\geq 30 \mathrm{~min}$ ) (table 5). Similarly, scores from the maternal EPDS attained when the infant was 4 months of age and adjusted for baseline EPDS score were the same across groups. Maternal fatigue produced mixed results at different time points, with any differences observed being too small to be of clinical importance. In addition, no differences in fatigue scores were apparent between those who received the sleep intervention (sleep and combination) and those who did not (FAB and control).

\section{DISCUSSION}

Within this randomised controlled study, a brief sleep intervention consisting of antenatal education and a 3-week home visit was not effective in preventing infant sleep problems, when compared with standard care. A key education target was to encourage infants to self-settle to sleep. ${ }^{45}$ There was a tendency towards those groups who received the sleep intervention being more likely to put their baby down to sleep awake, but the differences were not statistically significant $(p=0.052)$. We found some evidence that the sleep intervention influenced actigraphy-derived infant sleep patterns by significantly reducing the number of night wakings and lengthening daytime sleep, but the small size of the differences encountered means they are unlikely to be clinically significant. Parental sleep, fatigue and parental symptoms of depression were not affected by the interventions.

Twenty per cent of mothers reported that their 6-month-old infant's sleep was problematic for them, which is similar to other studies reporting a prevalence of $16 \%-38 \%$ within the first year of life. ${ }^{1-3}$ Parent-rated problematic sleep is a strong predictor of frequent and prolonged night wakings and difficulty settling to sleep. ${ }^{46}{ }^{47}$ Here we also collected data on partners (mostly fathers), and almost as many partners (17\%) as mothers $(20 \%)$ reported their child's sleep was a problem for them at 6 months of age, although there was significant discordance in mother-partner pairs. Few studies report children's problematic sleep from the partners' or fathers' perspective, but there is evidence suggesting a link between sleep problems in 4-year to 5-year-old children and poorer paternal general health. ${ }^{10}$

There was limited room for improvement for some behaviours. For example, at 6 months, more than two-thirds of the parents who were in a group who did not receive the sleep intervention frequently practised behaviours considered to be key intervention targets, such as putting their baby to sleep awake or letting their baby fall asleep without a parent being present. Approximately $80 \%$ of mothers reported that they could frequently recognise when their infant was tired, suggesting this may be an ineffective target to achieve change in this study population. However, we could not objectively measure the extent to which parents recognised the signs of tiredness, nor their ability to recognise the signs early enough to intervene effectively.

Although there are few studies to compare our data with directly, some target behaviours were more commonly practised than those reported in a similar Australian RCT $^{2}$ with follow-up also at 6 months of age. In that study, $11 \%$ of parents in the control group consistently allowed their baby to fall asleep without being present (a 
Table 3 Infant sleep diary and actigraphy data at 6 months of age

top ranking of 5 on a $1-5$ point scale of frequency). In our study $26 \%$ of parents in the control group rated this behaviour with a top ranking of 7 on a 1-7 point scale of frequency. Similarly, in the Australian study, ${ }^{2} 23 \%$ stated they could always recognise tired signs in their infant (top ranking 5) compared with our 44\% (top ranking 7). These data perhaps reflect that mothers in our study were well educated, with $65 \%$ having a university degree and with free access to a strong Well Child support service offered at multiple time points across the first 6 months of life. ${ }^{35}$ More closely aligned was the prevalence of settling baby to sleep while awake: $33 \%$ of control children in the Australian study ${ }^{2}$ compared with $27 \%$ in our study gave this the top ranking.

Four large RCTs have employed similar preventive strategies with a similar number of contact points ${ }^{27-29}$ (although intervening immediately after birth or up to 3 months of age). The outcomes of these studies and the current one lead us to suggest that preventive strategies may only confer benefit when delivered beyond the antenatal or immediate newborn period, that is, once parents have had time to understand their infants' sleep behaviours and patterns. One RCT intervening immediately after birth in primiparous women found no differences in infant sleep or maternal outcomes at follow-up ( 6 and 12 weeks postpartum), ${ }^{28}$ whereas three RCTs intervening at 2-3 weeks, ${ }^{29} 4$ weeks ${ }^{2}$ and 3 months postpartum ${ }^{27}$ resulted in longer infant sleep, ${ }^{2}$ fewer symptoms of maternal depression, ${ }^{2}$ less infant crying, ${ }^{2} 29$ and fewer infants with sleep problems, ${ }^{2}$ settling difficulties and night wakings ${ }^{27}$ at follow-up. Similar positive outcomes have been found in other study designs (non-RCTs) employing preventive strategies and when the interventions were delivered at a later time point (4 months of age). ${ }^{48} 49$ Other small trials with several more follow-up contacts have focused on preventive strategies in target groups, for example, primiparous women ${ }^{5051}$ or mothers of breastfed infants, ${ }^{51}$ reporting some successes with infants 'sleeping through the night' earlier ${ }^{51}$ and reduced parental wakings and enhanced stability of early but not later sleep patterns. ${ }^{50}$ As suggested by others, increasing the 'dose' of intervention may be important as may be targeting high-risk infants in more need of sleep interventions. ${ }^{28}$

The additional antenatal and postnatal education around safe sleep practices provided by the POI study made no difference to practice, but again the ceiling for improvement was limited. Some have suggested that safe sleep education should be started during pregnancy 
Table 4 Parent-rated sleep problems and sleep education-related outcomes from questionnaire at 4 and 6 months

$p$ Value for

intervention

\begin{tabular}{|c|c|c|c|c|c|c|c|c|}
\hline Outcome & $\begin{array}{l}\text { Infant } \\
\text { age } \\
\text { (months) }\end{array}$ & $\mathbf{n}$ & Control & FAB & Sleep & Combination & $\begin{array}{l}\Delta \text { Four } \\
\text { groups }\end{array}$ & $\begin{array}{l}\Delta \text { Two } \\
\text { groups* }\end{array}$ \\
\hline \multicolumn{9}{|l|}{ Infant sleep problem score ${ }^{\dagger \neq \S}$} \\
\hline \multirow[t]{2}{*}{ Mother-rated } & 4 & 718 & $2.0(1.9)$ & $2.3(1.9)$ & $2.2(1.9)$ & $2.0(2.0)$ & & \\
\hline & 6 & 701 & $2.2(2.0)$ & $2.6(1.9)$ & $2.4(2.0)$ & $2.3(1.9)$ & 0.176 & 0.961 \\
\hline \multirow[t]{2}{*}{ Partner-rated } & 4 & 469 & $1.9(1.8)$ & $2.3(1.8)$ & $2.3(1.8)$ & $2.2(1.8)$ & & \\
\hline & 6 & 428 & $2.3(1.8)$ & $2.5(1.9)$ & $2.4(1.8)$ & $2.3(1.7)$ & 0.117 & 0.410 \\
\hline \multirow[t]{2}{*}{ Infant unsettled, n (\%) } & 4 & 711 & $11(5.7)$ & $16(9.0)$ & $11(6.4)$ & $11(6.5)$ & & \\
\hline & 6 & 700 & $15(8.0)$ & $15(8.3)$ & $11(6.6)$ & $7(4.2)$ & 0.557 & 0.375 \\
\hline \multirow[t]{2}{*}{ Sleep latency $\geq 30 \mathrm{~min}, \mathrm{n}(\%)$} & 4 & 715 & $10(5.2 \%)$ & $10(5.6 \%)$ & $16(9.3 \%)$ & $14(8.2 \%)$ & & \\
\hline & 6 & 701 & $18(9.6 \%)$ & $15(8.3 \%)$ & $22(13.2 \%)$ & $5(3.0 \%)$ & 0.038 & 0.160 \\
\hline \multirow[t]{2}{*}{ Recognise tired signs score } & 4 & 717 & $6.1(0.9)$ & $6.0(0.9)$ & $6.1(0.9)$ & $6.1(0.9)$ & & \\
\hline & 6 & 700 & $6.2(0.8)$ & $6.1(0.8)$ & $6.1(0.9)$ & $6.3(0.8)$ & 0.166 & 0.805 \\
\hline \multirow[t]{2}{*}{ Infant put to bed awake score } & 4 & 717 & $5.1(1.8)$ & $5.1(1.8)$ & $5.4(1.7)$ & $5.4(1.8)$ & & \\
\hline & 6 & 700 & $5.5(1.8)$ & $5.2(1.8)$ & $5.5(1.8)$ & $5.6(1.6)$ & 0.129 & 0.052 \\
\hline \multirow[t]{2}{*}{ No parental contact score ${ }^{\Uparrow}$} & 4 & 717 & $5.0(1.8)$ & $5.0(1.7)$ & $5.3(1.9)$ & $5.0(2.0)$ & & \\
\hline & 6 & 700 & $5.3(1.8)$ & $5.1(1.9)$ & $5.3(1.9)$ & $5.1(2.0)$ & 0.444 & 0.389 \\
\hline \multirow[t]{2}{*}{ Bedsharing, n (\%) } & 4 & 720 & $11(5.6 \%)$ & $12(6.6 \%)$ & $12(7.0 \%)$ & $17(9.9 \%)$ & & \\
\hline & 6 & 685 & $11(6.0 \%)$ & $12(6.9 \%)$ & $7(4.1 \%)$ & $13(8.2 \%)$ & - & - \\
\hline \multirow[t]{2}{*}{ Smoking allowed at home, $\mathrm{n}(\%)$} & 4 & 717 & $4(2.1 \%)$ & $0(0.0 \%)$ & $0(0.0 \%)$ & $0(0.0 \%)$ & & \\
\hline & 6 & 687 & $1(0.5 \%)$ & $0(0.0 \%)$ & $0(0.0 \%)$ & $0(0.0 \%)$ & - & - \\
\hline \multirow[t]{2}{*}{$\begin{array}{l}\text { Smoking allowed in car when } \\
\text { children present, } \mathrm{n}(\%)\end{array}$} & 4 & 702 & $2(1.1 \%)$ & $3(1.7 \%)$ & $2(1.2 \%)$ & $0(0.0 \%)$ & & \\
\hline & 6 & 686 & $1(0.6 \%)$ & $1(0.6 \%)$ & $3(1.8 \%)$ & $3(1.8 \%)$ & - & - \\
\hline Sleeping on back, n (\%) & 4 & 718 & $176(90.3 \%)$ & $164(91.1 \%)$ & $152(88.9 \%)$ & $150(87.2 \%)$ & 0.631 & 0.254 \\
\hline Sheepskin use, n (\%) & 4 & 720 & 27 (13.9\%) & $17(9.4 \%)$ & $26(15.1 \%)$ & 27 (15.7\%) & 0.374 & 0.240 \\
\hline
\end{tabular}

Data presented as mean (SD) unless stated otherwise.

*'Sleep' (control and FAB combined) versus 'No Sleep' (sleep and combination combined).

†Secondary outcome to the main RCT. ${ }^{30}$ All others variables are additional outcomes.

fFrom a possible score of 1 (no problem) to 8 (large problem), thus higher score is better.

$\S$ Mean is geometric mean.

qFrom a possible score of 1 (never) to 7 (always), thus higher score is better.

-, Model not investigated due to small number of events not allowing estimating intervention effects with stratification variables included.

FAB, food, activity and breastfeeding intervention; RCT, randomised controlled trial. 
Table 5 Maternal and partner variables at 4 and 6 months

p Value for

intervention

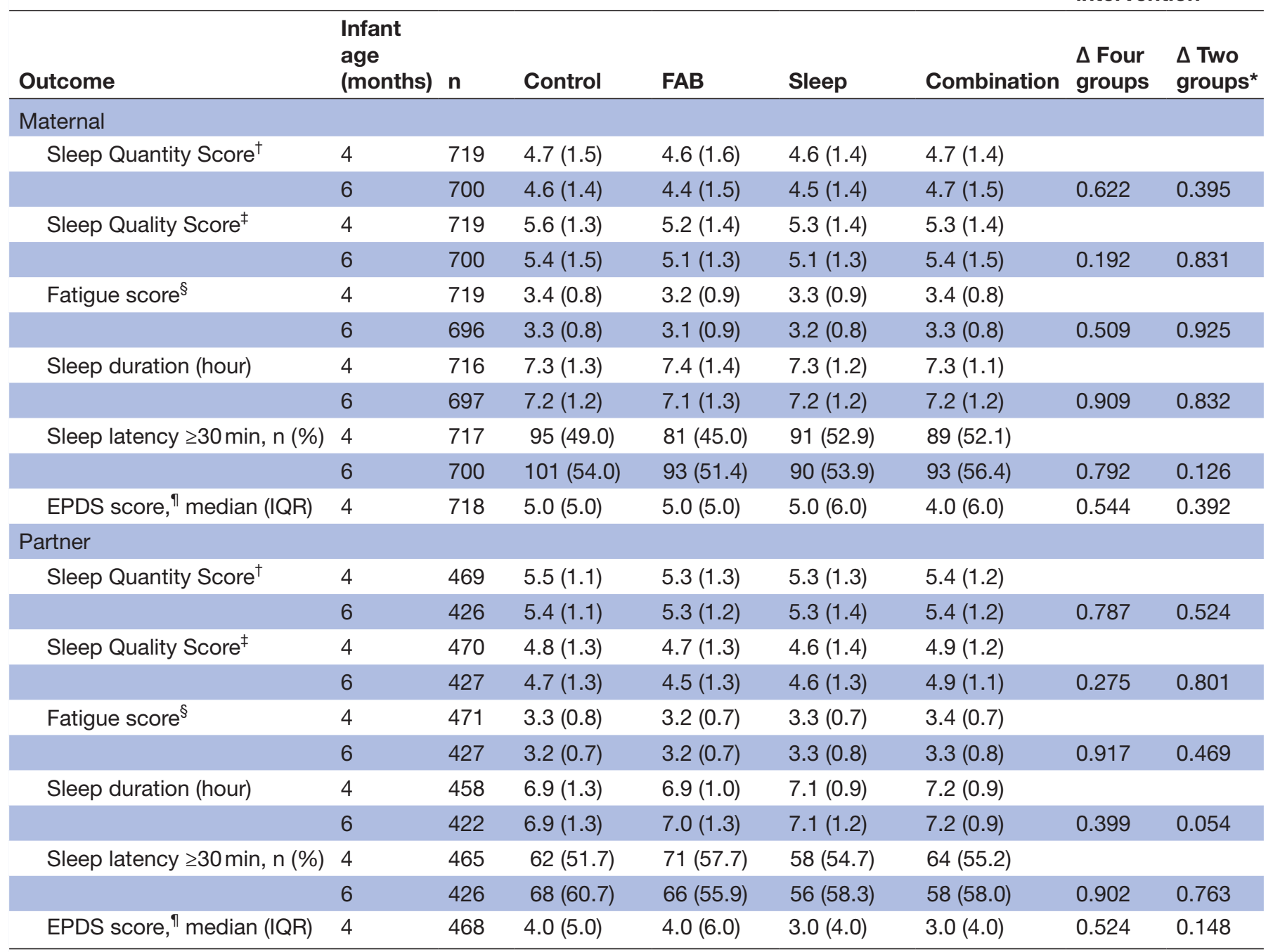

Data presented as mean (SD) unless stated otherwise. All variables are additional outcomes of the main $\mathrm{RCT}^{30}$

*'Sleep' (control and FAB combined) versus 'No Sleep' (sleep and combination combined).

†From a possible score of 1 (not nearly enough) to 8 (more than enough), thus higher score is better.

$\ddagger$ From a possible score of 1 (very bad) to 8 (very good), thus higher score is better.

$\S$ From a possible score of 1 (complete exhaustion) to 5 (well rested), thus higher score is better.

qEPDS, Edinburgh Postnatal Depression Scale (0-30); higher score is worse.

$\mathrm{FAB}$, food, activity and breastfeeding intervention; $\mathrm{RCT}$, randomised controlled trial.

and should be consistently reinforced throughout the first year. ${ }^{52} 53$ Our study data indicated that safe sleep messages are reaching our community, and further reinforcement of messages beyond standard Well Child care would confer no additional benefit, but we acknowledge that this may be important in other communities or cultures where unsafe sleep practices are more common.

Outcomes related to parental depression symptoms, although not significant, were important to consider given that sleep disturbance is considered a risk factor for postnatal depression, ${ }^{54}$ and a previous preventive strategy has been effective in reducing maternal depression symptoms. ${ }^{2}$ Maternal and partner subjective sleep quantity and quality, as well as symptoms of depression, were not influenced by the intervention. For mothers who have already been exposed to sleep disturbance during pregnancy, particularly within the last trimester, ${ }^{55}$ the naturally shorter sleep-wake patterns of infants can further contribute to their own sleep loss, with estimates of up to 1.5 hours of sleep lost over 24 hours in the early postnatal period. ${ }^{56}$ In the current study, self-reported usual overnight sleep duration was reduced approximately 1 hour on average from the third trimester to 4 months postpartum, and in partners by approximately 36 min over the same time period. However we did not collect data on daytime napping and therefore cannot ascertain if any night-time sleep loss was counterbalanced by daytime gain. 
The study had some important limitations. First, the results are limited to young infants in a well-educated community, thereby reducing the generalisability of the findings. Second, the sleep education was targeting some practices that were expected to be common practice, leaving relatively little room for improvement. However, the prevalence of sleep problems in our participants was just as high as elsewhere, suggesting there is still much benefit to be gained from Well Child or other services delivering sleep education. A different focus might be necessary for measurable gains, for example targeting more socially disadvantaged groups and focusing on parental sleep, where small gains have been observed. ${ }^{57}$ Furthermore, subjective reports of parental sleep practices related to our education targets, such as infant self-settling, may not be accurate enough, and more objective tools for measuring these are required. Study strengths include the randomised and controlled nature of the trial, high recruitment rates and retention, the longitudinal nature of the study, novel collection of data around parents' own sleep, and inclusion of the partner/ father.

In conclusion, the sleep intervention administered as a prevention strategy and conducted as part of an RCT in the POI study was not effective in preventing sleep problems in infants. The antenatal period may be too early to intervene successfully with sleep where the priority focus for families is on the impending birth. Finally, although our study, like so many others, focused on trying to achieve behaviour change through education, we acknowledge that alternative paradigms to preventing or treating sleep problems in infants have been suggested. In these alternative paradigms, infant night waking in the first 6 months is considered to be normal, and a range of strategies are used to help parents become more synchronised with their babies' sleep-wake needs and make decisions about what is right for them in their own unique situation with their own unique baby. ${ }^{58}$

Acknowledgements We thank the families and their infants who participated in this study. We also gratefully acknowledge the research assistance of Catherine Barker, Barbara Churcher, Rhondda Davies, Maha Hanna, Carmen Lobb, Michelle McGrath, Amelia Needs, Susan Peters, Nick Prosser, Megan Somerville and Bronwyn Thomas.

Contributors BJT, BCG and RMS led the sleep intervention, and RWT and ALMH led the FAB intervention. ARG designed and completed all statistical analyses and wrote the relevant sections of the manuscript. JAL coordinated and led the management of the study. All remaining authors contributed to writing the manuscript, study design and/or data collection. All authors had intellectual input into the manuscript, commented on drafts and approved the final version.

Funding This study was funded by the Health Research Council of New Zealand (Grant 08/374) and the Southern District Health Board. RWT is supported by the KPS Fellowship in Early Childhood Obesity. SLC was supported by a University of Otago Health Sciences postdoctoral fellowship. The funders had no role in study design; or in the collection, analysis and interpretation of data; or in the writing of the report or the decision to submit the article for publication.

Competing interests None declared.

Ethics approval The study was approved by the New Zealand Lower South Regional Ethics Committee (LRS/08/12/ 063), and all adult participants gave written informed consent on behalf of themselves and their babies.
Provenance and peer review Not commissioned; externally peer reviewed.

Data sharing statement № additional data are available.

Open Access This is an Open Access article distributed in accordance with the Creative Commons Attribution Non Commercial (CC BY-NC 4.0) license, which permits others to distribute, remix, adapt, build upon this work non-commercially, and license their derivative works on different terms, provided the original work is properly cited and the use is non-commercial. See: http://creativecommons.org/ licenses/by-nc/4.0/

(C) Article author(s) (or their employer(s) unless otherwise stated in the text of the article) 2017. All rights reserved. No commercial use is permitted unless otherwise expressly granted.

\section{REFERENCES}

1. Wake M, Morton-Allen E, Poulakis Z, et al. Prevalence, stability, and outcomes of cry-fuss and sleep problems in the first 2 years of life: prospective community-based study. Pediatrics 2006; $117: 836-42$.

2. Hiscock H, Cook F, Bayer J, et al. Preventing early infant sleep and crying problems and postnatal depression: a randomized trial. Pediatrics 2014;133:e346-54.

3. Armstrong KL, Quinn RA, Dadds MR. The sleep patterns of normal children. Med J Aust 1994;161:202-6.

4. Evanoo G. Infant crying: a clinical conundrum. J Pediatr Health Care 2007;21:333-8.

5. St James-Roberts I, Halil T. Infant crying patterns in the first year: normal community and clinical findings. J Child Psychol Psychiatry 1991;32:951-68.

6. Forsyth BW, Leventhal JM, McCarthy PL. Mothers' perceptions of problems of feeding and crying behaviors. A prospective study. Am J Dis Child 1985;139:269-72.

7. Touchette E, Côté SM, Petit D, et al. Short nighttime sleepduration and hyperactivity trajectories in early childhood. Pediatrics 2009;124:e985-93.

8. Hiscock H, Bayer J, Gold L, et al. Improving infant sleep and maternal mental health: a cluster randomised trial. Arch Dis Child 2007;92:952-8.

9. Giallo R, Rose N, Vittorino R, Fatigue VR. Fatigue, wellbeing and parenting in mothers of infants and toddlers with sleep problems. $J$ Reprod Infant Psychol 2011;29:236-49.

10. Martin J, Hiscock H, Hardy P, et al. Adverse associations of infant and child sleep problems and parent health: an australian population study. Pediatrics 2007;119:947-55.

11. Sadeh A, Tikotzky L, Scher A. Parenting and infant sleep. Sleep Med Rev 2010;14:89-96.

12. Touchette E, Petit D, Paquet J, et al. Factors associated with fragmented sleep at night across early childhood. Arch Pediatr Adolesc Med 2005;159:242-9.

13. Zuckerman B, Stevenson J, Bailey V. Sleep problems in early childhood: continuities, predictive factors, and behavioral correlates. Pediatrics 1987;80:664-71.

14. Kataria S, Swanson MS, Trevathan GE. Persistence of sleep disturbances in preschool children. J Pediatr 1987;110:642-6.

15. Pollock Jl. Night-waking at five years of age: predictors and prognosis. J Child Psychol Psychiatry 1994;35:699-708.

16. Turnbull K, Reid GJ, Morton JB. Behavioral Sleep problems and their potential impact on developing Executive function in Children. Sleep 2013;36:1077-84.

17. Touchette E, Petit D, Séguin JR, et al. Associations between sleep duration patterns and behavioral/cognitive functioning at school entry. Sleep 2007;30:1213-9.

18. Gruber R, Laviolette R, Deluca $P$, et al. Short sleep duration is associated with poor performance on IQ measures in healthy schoolage children. Sleep Med 2010;11:289-94.

19. Smedje H, Broman JE, Hetta J. Associations between disturbed sleep and behavioural difficulties in 635 children aged six to eight years: a study based on parents' perceptions. Eur Child Adolesc Psychiatry 2001;10:1-9.

20. Hiscock H, Canterford L, Ukoumunne OC, et al. Adverse associations of sleep problems in Australian preschoolers: national population study . Pediatrics 2007;119:86-93.

21. Carter PJ, Taylor BJ, Williams SM, et al. Longitudinal analysis of sleep in relation to BMI and body fat in children: the FLAME study. BMJ 2011;342:d2712.

22. Diethelm K, Bolzenius K, Cheng G, et al. Longitudinal associations between reported sleep duration in early childhood and the 
development of body mass index, fat mass index and fat free mass index until age 7. Int J Pediatr Obes 2011;6:e114-23.

23. Cappuccio FP, Taggart FM, Kandala NB, et al. Meta-analysis of short sleep duration and obesity in children and adults. Sleep 2008;31:619-26.

24. Hart CN, Jelalian E. Shortened sleep duration is associated with pediatric overweight. Behav Sleep Med 2008;6:251-67.

25. Kempler L, Sharpe L, Miller CB, et al. Do psychosocial sleep interventions improve infant sleep or maternal mood in the postnatal period? A systematic review and meta-analysis of randomised controlled trials. Sleep Med Rev 2016;29:15-22.

26. Hall WA, Hutton E, Brant RF, et al. A randomized controlled trial of an intervention for infants' behavioral sleep problems. BMC Pediatr 2015; $15: 181$

27. Kerr SM, Jowett SA, Smith LN. Preventing sleep problems in infants: a randomized controlled trial. J Adv Nurs 1996;24:938-42.

28. Stremler R, Hodnett E, Kenton L, et al. Effect of behaviouraleducational intervention on sleep for primiparous women and their infants in early postpartum: multisite randomised controlled trial. BMJ 2013;346:f1164.

29. Symon BG, Marley JE, Martin AJ, et al. Effect of a consultation teaching behaviour modification on sleep performance in infants: a randomised controlled trial. Med J Aust 2005;182:215-8.

30. Taylor BJ, Heath AL, Galland BC, et al. Prevention of Overweight in Infancy (POI.nz) study: a randomised controlled trial of sleep, food and activity interventions for preventing overweight from birth. BMC Public Health 2011;11:942.

31. Taylor BJ, Gray AR, Galland BC, et al. Targeting Sleep, Food, and activity in infants for Obesity Prevention: an RCT. Pediatrics 2017;139:e20162037.

32. Sadeh A, Anders TF. Infant sleep problems: origins, assessment, interventions. Infant Ment Health J 1993;14:17-34.

33. Van Cauter E, Holmback U, Knutson K, et al. Impact of sleep and sleep loss on neuroendocrine and metabolic function. Horm Res 2007;67(Suppl 1):2-9.

34. Salmond C, Crampton P, Atkinson J. NZDep2006 Index of Deprivation. Wellington: University of Otago, 2007.

35. Ministry of Health. Indicators for the Well Child/Tamariki Ora quality improvement framework-March 2014: Wellington, New Zealand: Ministry of Health, 2014.

36. Mindell JA, Telofski LS, Wiegand B, et al. A nightly bedtime routine: impact on sleep in young children and maternal mood. Sleep 2009;32:599-606.

37. Galland BC, Gray A, Sayers RM, et al. Safe sleep practices in a New Zealand community and development of a Sudden unexpected death in Infancy (SUDI) risk assessment instrument. BMC Pediatr 2014:14:263.

38. Cameron SL, Heath AL, Gray AR, et al. Lactation consultant support from late pregnancy with an Educational intervention at 4 months of Age Delays the introduction of complementary Foods in a Randomized Controlled Trial. J Nutr 2015;145:1481-90.

39. Cox JL, Holden JM, Sagovsky R. Detection of postnatal depression. development of the 10-item Edinburgh Postnatal Depression Scale. Br J Psychiatry 1987;150:782-6.
40. Galland B, Meredith-Jones K, Gray A, et al. Criteria for nap identification in infants and young children using 24-h actigraphy and agreement with parental diary. Sleep Med 2016;19:85-92.

41. Kahan BC, Morris TP. Improper analysis of trials randomised using stratified blocks or minimisation. Stat Med 2012;31:328-40.

42. Kahan BC. Bias in randomised factorial trials. Stat Med 2013:32:4540-9.

43. Murray D, Cox JL. Screening for depression during pregnancy with the edinburgh depression scale (EDDS). J Reprod Infant Psychol 1990;8:99-107.

44. Matthey S, Barnett B, Kavanagh DJ, et al. Validation of the Edinburgh Postnatal Depression Scale for men, and comparison of item endorsement with their partners. $J$ Affect Disord $2001 ; 64: 175-84$.

45. St James-Roberts I, Roberts M, Hovish K, et al. Video evidence that London Infants can resettle Themselves Back to Sleep after waking in the night, as well as Sleep for Long periods, by 3 months of Age. $J$ Dev Behav Pediatr 2015;36:324-9.

46. Hiscock H, Wake M. Infant sleep problems and postnatal depression: a community-based study. Pediatrics 2001;107:1317-22.

47. Sadeh A. A brief screening questionnaire for infant sleep problems: validation and findings for an internet sample. Pediatrics 2004:113:e570-77.

48. Adair R, Zuckerman B, Bauchner $\mathrm{H}$, et al. Reducing night waking in infancy: a primary care intervention. Pediatrics 1992;89:585-8.

49. Adachi $Y$, Sato $C$, Nishino $N$, et al. A brief parental education for shaping sleep habits in 4-month-old infants. Clin Med Res 2009;7:85-92.

50. Wolfson A, Lacks P, Futterman A. Effects of parent training on infant sleeping patterns, parents' stress, and perceived parental competence. J Consult Clin Psychol 1992;60:41-8.

51. Pinilla T, Birch LL. Help me make it through the night: behavioral entrainment of breast-fed infants' sleep patterns. Pediatrics 1993:91:436-44.

52. Ottolini MC, Davis BE, Patel K, et al. Prone infant sleeping despite the "Back to Sleep" campaign. Arch Pediatr Adolesc Med 1999:153:512-7.

53. Saririan S, Hauck FR. New recommendations to reduce the risk of SIDS: what should we advise parents? Am Fam Physician 2006;74:1839-40.

54. Armstrong KL, Van Haeringen AR, Dadds MR, et al. Sleep deprivation or postnatal depression in later infancy: separating the chicken from the egg. J Paediatr Child Health 1998;34:260-2.

55. Lee KA, Zaffke ME, McEnany G. Parity and sleep patterns during and after pregnancy. Obstet Gynecol 2000;95:14-18.

56. Signal TL, Gander PH, Sangalli MR, et al. Sleep duration and quality in healthy nulliparous and multiparous women across pregnancy and post-partum. Aust N Z J Obstet Gynaecol 2007;47:16-22.

57. Lee KA, Gay CL. Can modifications to the bedroom environment improve the sleep of new parents? two randomized controlled trials. Res Nurs Health 2011;34:7-19.

58. Whittingham $K$, Douglas $P$. Optimizing parent-infant sleep from birth to 6 months: a new paradigm. Infant Ment Health J 2014;35:614-23. 